\title{
MRI Findings in Patients with Symptoms of Trigeminal Neuralgia - A Retrospective Study
}

\author{
Shameem Ahamed $\mathbf{M}^{1}$, Rejeesh Saseendran ${ }^{2}$, Mohanan $\mathrm{K}^{3}$, Lisna Hameed PK ${ }^{4}$, Joy P Verghese ${ }^{5}$ \\ ${ }^{1}$ Assistant professor, Department of Radiodiagnosis, ${ }^{2}$ Assistant professor, Department of Radiodiagnosis, ${ }^{3}$ Professor and HOD, Department \\ of Radiodiagnosis, ${ }^{4}$ Junior Resident, Department of Radiodiagnosis, ${ }^{5}$ Associate Professor, Department of Radiodiagnosis, MES Medical \\ College, Perinthalmanna, Kerala India.
}

Corresponding author: Dr. Rejeesh Saseendran, Assistant Professor Department of Radiodiagnosis, MES Medical College, Perinthalmanna, Kerala India.

DOI: 10.21276/ijcmsr.2018.3.2.25

How to cite this article: Shameem Ahamed M, Rejeesh Saseendran, Mohanan K, Lisna Hameed PK, Joy P Verghese. MRI findings in patients with symptoms of trigeminal neuralgia - a retrospective study. International Journal of Contemporary Medicine Surgery and Radiology. 2018;3(2):B102-B105.

\section{A B S T R A C T}

Introduction: Trigeminal neuralgia (TGN) is an intense facial pain in the distribution of the trigeminal nerve. The common reason for TGN is the compression of the cisternal division of the trigeminal nerve by a vessel, commonly by an artery. Magnetic Resonance Imaging (MRI) plays an important and confirmatory role in demonstrating neuro vascular conflict which is the commonest causative factor for TGN. This study was conducted to evaluate the cases of facial pain in the distribution of trigeminal nerve by MRI to assess the pathology behind and to link the clinical data with imaging findings.

Material and methods: 47 patients with trigeminal pain with or without associated neurologic symptoms were included in this study. MRI of the brain, dedicated trigeminal nerve protocol (SPACE) was conducted with contrast injection in the selected cases according to the findings in the conventional scan. Causative assessment of TGN was conducted.

Results: Among 34 patients, MR could depict the etiology of trigeminal pain and 13 patients had idiopathic type TGN. In 27 patients, trigeminal nerves were seen in close proximity to vascular loops. In 16 patients the cisternal portion or root entry zones of nerves were significantly compressed due to vascular loops. In the majority of cases, the culprit vessel was a superior cerebellar artery. One patient had infarct involving the brain stem, one patient cavernoma involving pons and another one patient had features of gliosis in the region of the trigeminal nucleus in the brain stem. Extra-axial mass lesions compressing the trigeminal nerve were detected in four patients.

Conclusion: MRI can yield high accuracy in the evaluation and appropriate treatment of patients with TGN.

Key words: Trigeminal neuralgia, MRI, vascular loops, neuropathy, trigeminal nerve.

\section{INTRODUCTION}

The trigeminal nerve is the fifth of 12 pairs of cranial nerves in the head and is responsible for the main sensory perception of the face. ${ }^{1}$ Trigeminal neuralgia (TGN), or tic dolourex, is an unbearable condition consisting of unilateral short bursts of lancinating pain in the allocation of one or more branches of the trigeminal nerve. It is the most common facial neuralgia, explains the most excruciating pain known to humanity, happens in episodes and exclusively unilateral, with more chances towards the right side. ${ }^{2}$ The pain may cause due to the irritation of the trigeminal nerve, involves the lower face and jaw, sometimes it also affects the area around the nose and above the eye. The nature of pain could be like an electric shock, brief, intense, stabbing, and repeated in every few seconds; it can be triggered by several actions such as speaking, chewing, swallowing, brushing the teeth or touching the face.

Studies report that, in every year more than 150,000 people were diagnosed with trigeminal neuralgia with a prevalence of 3-6 per 1,00,000. ${ }^{3}$ The condition can occur at any age and sex, but most commonly diagnosed in adults in their 5 th to 7 th decades of life with more predictive value in female population. ${ }^{4}$ It is also reported to have a genetic risk factor, that the disorder runs in families due to an inherited blood vessel formation. Diseases like hypertension and multiple sclerosis (MS) act as risk factors for TGN. Irritation of the trigeminal nerve may lead to the pain, which may happen due to the contact of trigeminal nerve with artery or vein at the base of the brain. This lead to a misfire of nerves in the brain due to the pressure created on nerves. Other reasons such as intracranial tumours, demyelinating or inflammatory causes may also trigger the condition of TGN. ${ }^{5}$

There are several effective ways to alleviate the pain, including a variety of medications such as carbamazepine, phenytoin, muscle relaxants, gabapentin, clonazepam, sodium valproate, lamotrigine and topiramate. Open cranial surgery and lesioning procedures were also available for the management of pain associated with TGN. Before this, accurate diagnosis of cause for TGN may have an important role in the management of these conditions. The diagnosis of TGN is commonly based on the patient history and description of symptoms with the neurological and physical examinations. The other disorders which cause facial pain such as post- 
herpetic neuralgia, temporomandibular joint disorder and cluster headaches should be ruled out before TN is diagnosed. Obtaining a correct diagnosis is difficult due to the occurrence of overlapping symptoms and several reasons can cause facial pain. Hence the finding of the causative reason for facial pain could be helpful for the detection of TGN.

The visualization of the trigeminal nerve is possible by using modern imaging techniques. Magnetic resonance imaging (MRI) is the important and primary method for evaluating symptoms associated with a trigeminal nerve in the majority clinical settings. ${ }^{6}$ MRI can detect the reason for nerve disturbance such as a tumour or MS, which leads to TGN. In other cases, MR imaging of brain may help to find the reason for nerve irritation. Hence the study was carried to evaluate the MRI findings in patients with trigeminal neuralgia.

\section{MATERIAL AND METHODS}

A retrospective study was conducted from January to December 2017 in radiology and imaging department of MES Medical College after getting the approval of the ethical committee. Total of 47 patients with symptoms of trigeminal neuralgia who were referred to radiology and imaging department of MES Medical College for MR imaging were included in this study. Written informed consent was obtained from all patients. The study population consisted of 23 females and 24 males with an age range of 15 to 73 years. The inclusion criteria included patients with persistent facial pain \pm other neurological symptoms or signs, all patients underwent MRI and were assesses clinically by Neurologists. The exclusion criteria include any other associated cause of referred pain including dental troubles or referred cervical myalgia and if the patient has any general contraindication to MRI examination.

\section{MR Imaging}

All 47 patients underwent MR imaging in the standard protocol of MES Medical college hospital. The Imaging was performed with the use of a 1.5T MR imaging system (Siemens Avanto). Imaging sequences of the brain included Fast spin-echo T1-weighted images, T2-weighted images, heavily T2 weighted SPACE sequences, FLAIR and DWI. From DWI, ADC maps were calculated. SPACE sequence was performed in all patients to assess the trigeminal nerves at the root entry zones and cisternal portions. The contrastenhanced study was used in selected patients. Images were obtained from the level of brainstem nuclei to the extracranial branches of trigeminal nerves and muscles of mastication. Patients tolerated the examination with no complications.

\section{Images interpretation}

Two experienced radiologists retrospectively evaluated the MR images in consensus. Images were analyzed to look for probable causes of trigeminal neuralgia. Brain stem was carefully examined to look for any parenchymal lesions. Any vascular loops compressing trigeminal nerves were looked for in SPACE sequences. Intraaxial or extraaxial mass lesions were also looked for. Patients with normal MRI findings were recommended to do trigeminal tractography study for further evaluation of the trigeminal pathway.

\section{RESULTS}

The study population contains 47 patients having 24 males, 23 females with mean age of 39.67 years \pm 12.4 . Among them, 21 patients had trigeminal pain associated with other neurologic symptoms and/or signs (Fig. I), the other 26 patients presented with only trigeminal pain.

1.5 T MR studies were performed and the results of the

Associated neuralgic signs and symptoms

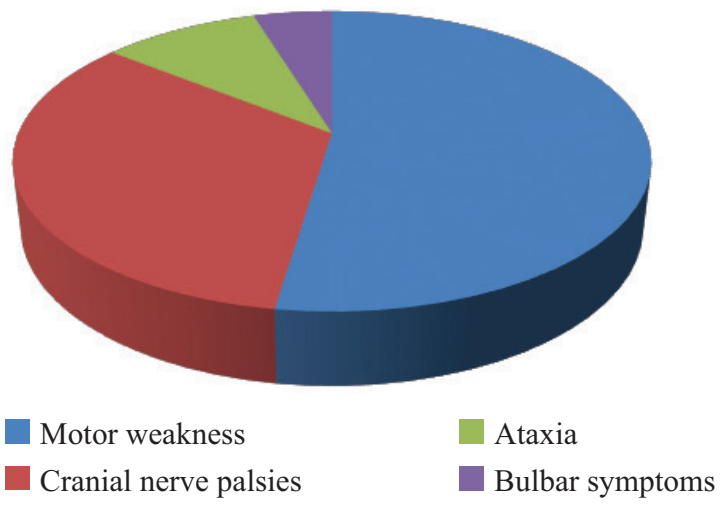

Figure-1: Associated neurologic signs and symptoms among the study population

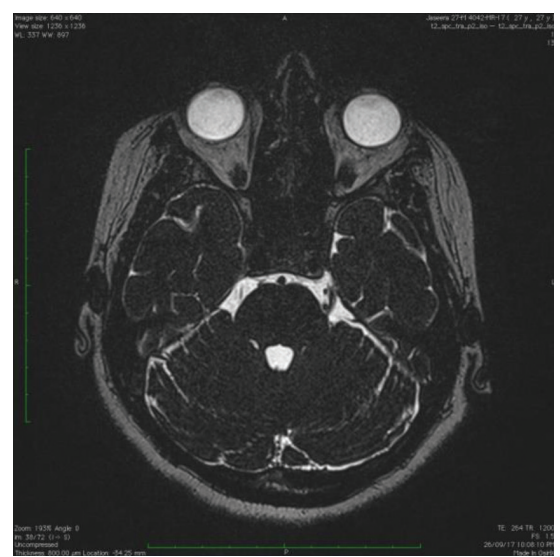

Figure-2: Axial SPACE sequence shows a loop of superior cerebellar artery impinging the root entry zone of left trigeminal nerve - neurovascular conflict.

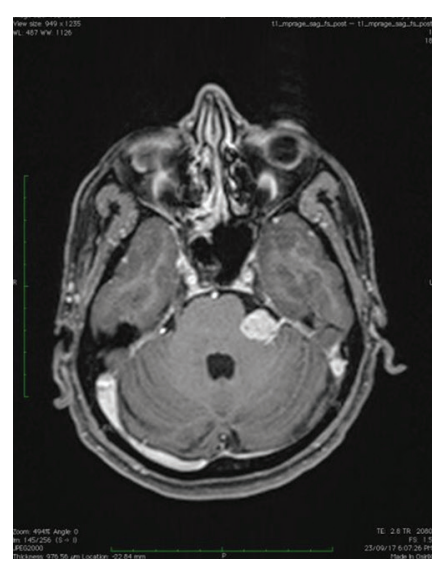

Figure-3: Axial post-contrast T1WFS image shows a strongly enhancing well-defined mass in the left cerebellopontine angle cistern, impinging the root entry zone of trigeminal nerve - suggestive of acoustic schwannoma. 


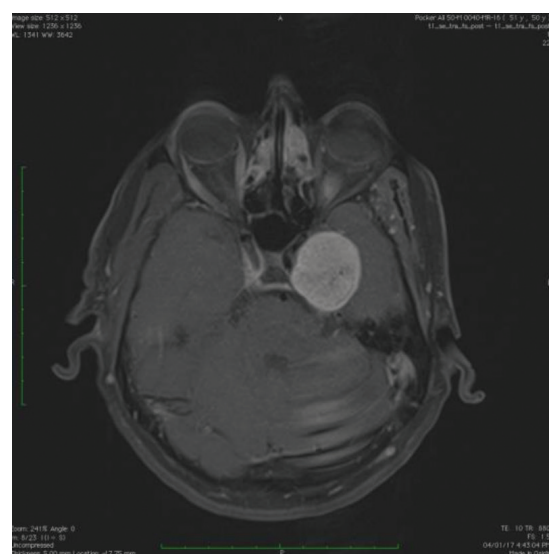

Figure-4: Axial post-contrast T1WFS image shows a strongly enhancing well-defined mass in the left Meckel's cave region - suggestive of trigeminal schwannoma.

clinical and imaging findings are summarized. Of the 47 patients who underwent MR imaging study was negative in 13 patients. Vascular loops in close proximity to trigeminal nerve were seen in 27 patients. In 9 patients loops were only abutting the nerve. Significant compression of root entry zones or a cisternal portion of nerves due to vascular loops were seen in 16 patients. The superior cerebellar artery was culprit vessel in the vast majority $(22$ cases) of patients (Fig. II). In one patient an AICA loop was seen abutting the trigeminal nerves bilaterally. In 4 patients exact origin of the vascular loop could not be made out and some appeared to be venous. One patient had infarct involving the brain stem. One patient showed features of gliosis, due to some previous insult, in the region of the trigeminal nucleus in the brain stem. One patient had cavernoma involving pons. In four patients extraaxial mass lesions were detected, compressing the trigeminal nerve - two acoustic schwannomas (Fig. III), one trigeminal schwannoma (Fig. IV) and one petroclival meningioma.

\section{DISCUSSION}

It is important to detect the etiology of facial pain, where the precise diagnosis is mandatory to plan the best and effective therapy. Several conditions like dental, ENT, and eye disorders may cause severe pain in the face; they can also miss considered as the primary head/facial pains. The International Association for the Study of Pain defines TGN as sudden, usually unilateral, severe, brief, stabbing, and recurrent episodes of pain in the distribution of one or more branches of the Vth nerve.

In this present study, 13 patients (27\%) were having the idiopathic type of trigeminal neuralgia as they were not found to have any structural abnormality in the MR imaging studies. This result was in accordance with Eman and McMillan, they reported that many cases of TGN can be due to the idiopathic reason, and in sometimes it may occur due to secondary conditions such as multiple sclerosis or tumours which can be imaged by CT or MRI., Even though DTI results are beyond the scope of our study, patients were suggested for additional examination such as MR tractography to study the trigeminal pathways.

Compressions of nerves by vessels are the major cause of
TGN. Most of the time the cause for compression may cause by a tortuous, elongated superior cerebellar artery (60-90\%). ${ }^{9}$ Vertebrobasilar dolichoectasia, elongated anterior inferior cerebellar artery or venous compression may found in fewer cases. Several autopsy studies show that $90 \%$ of the patient having TGN has some degree of contact between the 5 th nerve and a blood vessel. ${ }^{10}$ In the present study, 16 patients (34\%) were found to have a significant vascular loop compromising the trigeminal nerve roots. The compression was found in root entry zones or a cisternal portion of nerves. In 27 patients, vascular loops were in close proximity to trigeminal nerve and in 9 patients loops were only abutting the nerve. This compression and displacement of the nerve by the vascular loop is well evaluated by the CISS sequence, which demonstrates the thinning of the root entry zone, and allows exact identification of the vascular loop. It has been proposed as the initial screening procedure for all patients with refractory trigeminal neuralgia, especially if surgical intervention is being considered. ${ }^{11}$

The superior cerebellar artery was culprit vessel in the vast majority of patients. The neurovascular confliction of TGN patients is relatively complicated, and a superior cerebellar artery (SCA) along the shoulder of the REZ compressing the caudal side of the trigeminal nerve ventromedially is common. ${ }^{12,13}$ In our study, one patient had seen with an anterior inferior cerebellar artery (AICA) loop abutting the trigeminal nerves bilaterally. This was in accordance with other studies. ${ }^{14,15}$

The possibility of multiple offending vessels (arterial and/ or venous loops) should be excluded with careful inspection. In our series, venous compression is rare in $\mathrm{TN}$ patients. In 4 patients exact origin of the vascular loop could not be made out and a few appeared to be venous. One patient had infarct involving the brain stem. One patient showed features of gliosis, due to some previous insult, in the region of the trigeminal nucleus in the brain stem. Gliosis is the focal proliferation of glial cells in the CNS in response to insult. By strict definition, gliosis is not synonymous with encephalomalacia which is the end result of liquefactive necrosis of brain parenchyma following an insult. One patient had cavernomas involving pons, which was also reported in other studies. ${ }^{16,17}$

Intracranial extra-axial pathologies arise from tissues other than brain parenchymas, such as meninges, dura, calvarium, ventricles, choroid plexus, pineal gland, or pituitary gland. The present study shows extra-axial mass lesions in four patients, which compressing the trigeminal nerve.

There are many kinds of extra-axial tumours and tumourlike lesions, and their definitive diagnosis can often be made easily via imaging studies. In the present study, extra-axial tumours were two acoustic schwannomas, one trigeminal schwannoma and one petroclival meningioma. ${ }^{18,19}$

\section{CONCLUSION}

In patients with trigeminal neuropathy, clinical features alone may not be enough to localize the lesions in most cases. MR imaging is a non-invasive technique devoid of ionizing radiation, which allows multiplanar image acquisition. When properly performed and interpreted MR imaging can yield 
high accuracy in the evaluation and appropriate treatment of patients with trigeminal neuropathy. For this, images should be obtained from the level of nuclei in the brain stem to the extracranial branches of the nerve. SPACE or CISS sequences are very helpful in this regard and whenever needed contrast study should be performed.

\section{ACKNOWLEDGEMENT}

Authors are thankful to Mr Jaleel and Medi research direct for their support during the entire project.

\section{REFERENCES}

1. Woolsey TA, Hanaway J, Gado MH. The brain atlas: a visual guide to the human central nervous system. John Wiley and Sons 2017; 54(4): 284.

2. Devor M,Wood I, Sharav Y,Zakrzewska JM.Trigeminal neuralgia during sleep. Pain Practice 2008; 8(4): 263-8.

3. MacLean R, Fernandes T. Multiple sclerosis: understanding a complex neurological condition. Nursing Standard 2010; 24(28): 50

4. Koopman JS, Dieleman JP, Huygen FJ, de Mos M, Martin CG, Sturkenboom MC. Incidence of facial pain in the general population. PAIN. 2009; 147(1-3):122-7.

5. Sabalys G, Juodzbalys G, Wang HL. Aetiology and pathogenesis of trigeminal neuralgia: a comprehensive review. Journal of oral and maxillofacial research 2012;3(4)

6. C.B. Majoie, B. Verbeeten Jr, J.A. Dol, F.L. PeetersTrigeminal neuropathy: evaluation with MR imaging. Radiographics 1995; 15(5): 795-811.

7. Eman, Heba, Walid, Mohamed. Trigeminal pain: Potential role of MRI. The Egyptian Journal of Radiology and Nuclear Medicine 2016: 47 (4); 15491555.

8. J.M1. Zakrzewska, R. McMillan. Trigeminal neuralgia: the diagnosis and management of this excruciating and poorly understood facial pain. Postgrad Med J 2011; 87 (1028): 410- 416.

9. C.B.L.M. Majoie, F.J.H. Hulsmans, J.A. Castelijns, B. Verbeeten Jr, D. Tiren, E.J.R. van Beek, et al. Symptoms and signs related to the trigeminal nerve: diagnostic yield of MR. Radiology1998; 209(6): 557-562.

10. Miller JP, Acar F, Hamilton BE, Burchiel KJ. Radiographic evaluation of trigeminal neurovascular compression in patients with and without trigeminal neuralgia. Journal of neurosurgery 2009; 110(4):627-32.

11. M. Becker, R. Kohler, M.I. Vargas, M. Viallon, J. Delavelle. Pathology of the trigeminal nerve. Neuroimag Clin N Am 2008; 18(5): 283-307.

12. Oesman C, Mooij JJ. Long-term follow-up of microvascular decompression for trigeminal neuralgia. Skull Base. 2011; 21(3): 313.

13. Jagannath PM, Venkataramana NK, Bansal A, Ravichandra M. Outcome of microvascular decompression for trigeminal neuralgia using autologous muscle graft: a five-year prospective study. Asian J Neurosurg. 2012; 7(3): 125.

14. Kawashima M, Yamada M, Sato S, Oka H, Fujii K, Matsushima T. Hemifacial spasm caused by vascular compression of the distal portion of the facial nerve associated with configuration variation of the facial and vestibulocochlear nerve complex. Turk Neurosurg. 2009; 19(3): 269-75.

15. Polo G, Fischer C, Sindou MP, Marneffe V. Brainstem auditory evoked potential monitoring during microvascular decompression for hemifacial spasm: intraoperative brainstem auditory evoked potential changes and warning values to prevent hearing lossprospective study in a consecutive series of 84 patients. Neurosurgery. 2004; 54(5):97.

16. Bullitt E, Tew JM, Boyd J. Intracranial tumor in patient with facial pain. J Neurosurg. 1986; 64(2): 865-71.

17. Cambruzzi E, Presa K, Silveira LC, Perondi GE. Epidermid cyst of the posterior fossa. A case report. J Bras Pathol Med Lab. 2011; 47(4): 79-82.

18. B. Cirak, N. Kiymaz, A. ArslanogluTrigeminal neuralgia caused by intracranial epidermoid tumor: report of a case and review of the different therapeutic modalities. Pain Physician 2004;7 (1): 129-132.

19. H.B. Klieb, B.V. FreemanTrigeminal neuralgia caused by intracranial epidermoid tumour: report of a case. J Can Dent Assoc 2008; 74 (1): 63-65.

Source of Support: Nil; Conflict of Interest: None 